\title{
Glucose control during cardiac surgery: How sweet it is
}

\author{
Michael E. Jessen, MD
}

\author{
See related article on page \\ 1007.
}

From the Department of Cardiovascular and Thoracic Surgery, University of Texas Southwestern Medical Center at Dallas, Dallas, Tex.

Received for publication Dec 3, 2002; revisions requested Dec 9, 2002; revisions received Dec 16, 2002; accepted for publication Jan 6, 2003.

Address for reprints: Michael E. Jessen, MD, Department of Cardiovascular and Thoracic Surgery, University of Texas Southwestern Medical Center at Dallas, 5323 Harry Hines Blvd, Dallas, TX 75390-8879 (E-mail: michael.Jessen@ utsouthwestern.edu).

J Thorac Cardiovasc Surg 2003;125:985-7

Copyright () 2003 by The American Association for Thoracic Surgery

$0022-5223 / 2003 \$ 30.00+0$

doi: $10.1067 / \mathrm{mtc} .2003 .454$

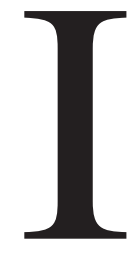

$\mathrm{t}$ has long been recognized that patients with diabetes pose a particular challenge to cardiologists and cardiovascular surgeons. Patients with diabetes have worse outcomes after acute myocardial infarction, ${ }^{1}$ have a more ominous outlook if congestive heart failure develops, and appear to be at increased risk for serious arrhythmias and sudden cardiac death. ${ }^{2}$ Compounding these problems is the fact that patients with diabetes may have more advanced, diffuse coronary disease, making interventional procedures or coronary artery bypass grafting (CABG) unattractive, or they may have end-organ renal or neurologic dysfunction that excludes them from other therapies, such as cardiac transplantation. Outcomes of patients with diabetes who undergo surgical revascularization have been shown to be inferior to those of their counterparts without diabetes. ${ }^{3}$ Any strategy that could improve outcomes in this group of patients would be a welcome addition to our surgical armamentarium.

In this issue of the Journal, Furnary and colleagues ${ }^{4}$ present a study of insulin management in patients who had undergone CABG. During a 15-year period, their management of glucose levels was altered from a protocol of intermittent subcutaneous insulin injections to one of continuous intravenous insulin infusion. Another important evolution was in the target serum glucose levels for these patients, a value that moved from $200 \mathrm{mg} / \mathrm{dL}$ to $100 \mathrm{mg} / \mathrm{dL}$ during the study interval. Associated with these changes in glucose and insulin management were significant improvements in outcomes. Operative mortality was significantly reduced, with the principal benefit accounted for by an improvement in cardiac-related mortality. Furnary and colleagues ${ }^{4}$ theorize that the mechanism of this effect is related to improved glucose uptake and use by the heart, leading to a superior metabolic condition within the myocyte and subsequent improved postischemic cardiac performance.

The concept that glucose and insulin management are important in myocardial ischemia is not new. More than 40 years have elapsed since the report of SodiPollares and associates ${ }^{5}$ describing a beneficial effect of an intravenous infusion of glucose, insulin, and potassium (GIK) on the electrophysiologic signs of myocardial infarction. A large number of experimental studies and clinical trials have been published since then, many showing a reduction in infarct size and improved survival in patients treated with this "metabolic cocktail." Not all studies of GIK have shown a benefit, however, and the use of this treatment modality is far from universal today. One feature that may contribute to these conflicting results is the use of different GIK regimens. To achieve meaningful metabolic results, a mixture of $30 \%$ glucose, 50 units of insulin, and $80 \mathrm{mmol}$ of potassium delivered at a rate greater than $1.5 \mathrm{~mL} /(\mathrm{kg} \times \mathrm{h})$ may be required. ${ }^{6}$ In addition, it should be noted that the vast majority of GIK studies have involved patients without diabetes. Patients with diabetes have impaired glucose uptake and increased plasma levels of free fatty acids during ischemia, conditions that increase their susceptibility to myocardial damage after ischemia. The Diabetes Insulin-Glucose in Acute Myocardial Infarction trial is the largest study to date to examine the effect of a similar metabolic strategy in patients with diabetes. It found a significant benefit of an insulin-glucose infusion as an adjunctive therapy for acute myocardial infarction. ${ }^{7}$ The rationale for further study of insulin therapy in patients with diabetes who undergo CABG therefore seems particularly timely.

It may seem intuitively obvious that better glucose management can improve the outcomes of patients with diabetes undergoing CABG. Latham and colleagues ${ }^{8}$ have shown that tighter glycemic control is associated with a reduction in wound 
infections after CABG, and higher glucose levels have been suggested to have an adverse effect on central nervous system morbidity after surgical revascularization. This study by Furnary and colleagues ${ }^{4}$ did not specifically address these postoperative complications, although this group has previously reported a reduction in deep sternal wound infections with their continuous intravenous insulin protocol. ${ }^{9}$ They did identify a significant mortality benefit that appeared to be related to cardiac events after surgery, in particular low cardiac output and left ventricular failure. As a result, Furnary and colleagues ${ }^{4}$ have concluded that tighter perioperative glucose management reduced mortality through improvements in myocardial metabolism after ischemia.

Abundant experimental data have accumulated to support this hypothesis. Insulin leads to increased glucose uptake by the heart, providing more substrate for glycolytic adenosine triphosphate (ATP) production during ischemia. Insulin may also increase pyruvate dehydrogenase activity, thereby improving oxidation of glucose and lactate during reperfusion and suppressing oxidation of fatty acids, which can be harmful to the postischemic heart. A significant correlation between glucose levels and mortality, as observed in this study, would seem to support this concept. However, the idea that altering insulin management and controlling hyperglycemia will improve results of CABG through this metabolic mechanism is not established by these results. The study provides an intriguing concept, but further investigations will be needed before we can completely understand why mortality is reduced with this protocol.

First, we need to gain a much greater understanding of the fate of glucose within the myocyte. Under normal conditions, glucose plays a relatively minor role in myocardial energy metabolism. Fatty acids are the major oxidative fuel used by the heart, accounting for $60 \%$ to $100 \%$ of myocardial oxygen consumption, with much lesser contributions from lactate and glucose. During ischemia, catabolism of glucose through the glycolytic pathway yields a relatively small amount of ATP compared with complete oxidation through the citric acid cycle, yet glycolytically derived ATP may have special significance. Experimental evidence suggests that glycolytically derived ATP is preferentially used for calcium reuptake by sarcoplasm reticulum, an essential process for diastolic relaxation. ${ }^{10}$ Glycolytically derived ATP preferentially inhibits cardiac ATP-sensitive potassium channels and is important for the optimal function of the sodium-potassium ATPase to prevent intracellular sodium accumulation during ischemia. Under aerobic conditions, manipulations that increase oxidation of glucose will increase acetyl coenzyme A levels. This can lead to a reciprocal decrease in oxidation of fatty acids, probably from an increase in malonyl coenzyme A production with a subsequent inhibition of carnitine palmityl transferase 1 activity. Strategies that switch cardiac metabolism from fatty acids to carbohydrate fuels are usually found to have beneficial effects on postischemic recovery.

But do these metabolic events occur in cardiac surgical patients treated with insulin, as outlined by Furnary and colleagues? ${ }^{4}$ We simply do not have enough information to know. Furnary and colleagues ${ }^{4}$ have not provided any data on levels of insulin, free fatty acids, intracellular metabolites, myocardial ATP levels, enzyme activity levels, or glycolytic rates. As a result, we must be cautious in attributing the beneficial effects to primary alterations in cardiac metabolism. Most of the experimental work defining the effects of glucose and insulin has been done on animal models that were not diabetic, making assumptions about mechanisms even more speculative.

Metabolism in the heart is altered by ischemia, and during ischemia glucose metabolism is slowed and confined to anaerobic (glycolytic) pathways. Although glycolytically derived ATP may be beneficial during ischemia under some experimental conditions, ultimately this process will lead to lactate accumulation and progressive intercellular acidosis. Neely and Grotyohann ${ }^{11}$ have shown that depletion of glycogen before myocardial ischemia is actually protective, presumably by reducing glucose metabolism and lactate accumulation. Thus glucose loading and subsequent glucose use during ischemia may be beneficial or deleterious, depending on the particular conditions encountered. In patients undergoing heart surgery, we rarely have enough information of their metabolic state to postulate whether an intervention that is dependent on these factors will lead to successful, deleterious, or indifferent outcomes.

The authors of this study in the Journal show a strong relationship between lower serum glucose levels (tighter glucose control) and mortality during a 15 -year period. The implication here is that improved insulin protocols move glucose into the heart cells to beneficially alter cardiac metabolism. Again, we must be cautious with this interpretation. Insulin administration results in reduction of blood glucose levels primarily by movement of glucose into tissues other than the heart. More than 30 years ago CurtisPrior and colleagues ${ }^{12}$ measured the contributions of different organs and tissues of the rat to the assimilation of glucose. The vast majority of glucose was taken up by skeletal muscle, skin, adipose tissue, and liver. Only a small fraction (less than $1 \%$ ) was taken up by the myocardium. ${ }^{12}$ In addition, patients with diabetes may have impaired myocardial glucose uptake, further skewing the relationship between serum glucose levels and cardiac metabolism. Recent studies have suggested that the relationship of administered insulin, cardiac glucose uptake, and ischemic tolerance may be even more complex. One recent report found that insulin-mediated cardioprotection was independent of 
the presence of glucose at reperfusion. ${ }^{13} \mathrm{McNulty}^{14}$ has shown that any increase in myocardial glucose extraction in response to systemic insulin is mediated as much by a reduction in circulating free fatty acids as by direct insulin action on the heart itself. Perhaps we should be monitoring free fatty acid levels instead of glucose levels in these patients.

Finally, the method of myocardial protection used for the patients in this study was intermittent fibrillation without the use of cardioplegia. This is a distinctly uncommon mode of myocardial protection for most surgeons today, and one that may more closely approximate the global ischemic models on which the potential metabolic mechanism is based. Surgeons who incorporate hypothermia or potassium cardioplegia into their myocardial protective techniques may already be achieving some of the metabolic effects that are attributed to tighter insulin control, because both hypothermia ${ }^{15}$ and potassium arrest ${ }^{16}$ have been experimentally demonstrated to alter myocardial substrate metabolism by suppressing fatty acid oxidation. Those who use warm continuous cardioplegia or off-pump techniques may be further limiting episodes of myocardial ischemia and as a result may not derive benefits of the magnitude observed in this study.

Nevertheless, the results presented by Furnary and colleagues ${ }^{4}$ for patients with diabetes are excellent and intriguing. Surgeons may find their insulin protocol to be a useful guide to incorporate in their own practices for these challenging patients. More work will be needed to elucidate the underlying mechanisms behind their observations. As we learn more about the metabolic consequences of insulin on postischemic cardiac glucose and fatty acid oxidation, we should be able to generate refinements in myocardial protective strategies that can take advantage of these mechanisms. This should ultimately lead to further improvements in the results of CABG for patients with diabetes. And that may be the sweetest result of all.

\section{References}

1. Smith JW, Marcus FI, Serokman R. Prognosis of patients with diabetes mellitus after acute myocardial infarction. Am J Cardiol. 1984;54: 718-21.
2. McNally PG, Lawrence IG, Panerai RB, Weston PJ, Thurston H. Sudden death in type 1 diabetes. Diabetes Obes Metab. 1999;1: 151-8.

3. Thourani VH, Weintraub WS, Stein B, Gebhart SS, Craver JM, Jones EL, et al. Influence of diabetes mellitus on early and late outcome after coronary artery bypass grafting. Ann Thorac Surg. 1999;67:1045-52.

4. Furnary AP, Gao G, Grunkemeier GL, Wu Y, Zerr KJ, Bookin SO, et al. Continuous insulin infusion reduces mortality in patients with diabetes undergoing coronary artery bypass grafting. J Thorac Cardiovasc Surg. 2003;125:1007-21.

5. Sodi-Pollares D, Testelli MD, Fishleder BL, Bisteni A, Medrano GA, Freidland C, et al. Effects of an intravenous infusion of a potassiumglucose-insulin solution on the electrophysiologic signs of myocardial infarction. Am J Cardiol. 1962;9:166-81.

6. Rogers WJ, Stanley AW, Breinig JB, Prather JW, McDaniel HG, Moraski RE, et al. Reduction of hospital mortality rate of acute myocardial infarction with glucose-insulin-potassium infusion. Am Heart J. 1976;92:441-54.

7. Malmberg K, Ryden L, Efendic S, Herlitz J, Nicol P, Waldenstrom A, et al. Randomized trial of insulin-glucose infusion followed by subcutaneous insulin treatment in diabetic patients with acute myocardial infarction (DIGAMI study): effects on mortality at 1 year. J Am Coll Cardiol. 1995;26:57-65.

8. Latham R, Lancaster AD, Covington JF, Pirolo JS, Thomas CS. The association of diabetes and glucose control with surgical-site infections among cardiothoracic surgery patients. Infect Control Hosp Epidemiol. 2001;22:607-12.

9. Furnary AP, Zerr KJ, Grunkemeier GL. Continuous intravenous insulin infusion reduces the incidence of deep sternal wound infection in diabetic patients after cardiac surgical procedures. Ann Thorac Surg. 1999;67:352-62.

10. Jeremy RW, Koretsune Y, Marban E, Becker LC. Relationship between glycolysis and calcium homeostasis in postischemic myocardium. Circ Res. 1992;70:1180-90.

11. Neely JR, Grotyohann LE. Role of glycolytic products in damage to ischemic myocardium. Circ Res. 1984;55:816-24.

12. Curtis-Prior PB, Trethewey J, Stewart GA, Hanley T. The contribution of different organs and tissues of the rat to assimilation of glucose. Diabetologia. 1969;5:384-91.

13. Jonassen AK, Sack MN, Mjos AD, Yellon DM. Myocardial protection by insulin at reperfusion requires early administration and is mediated via Akt and p70s6 kinase cell-survival signaling. Circ Res 2001;89: 1191-8.

14. McNulty PH. Comparison of local and systemic effects of insulin on myocardial glucose extraction in ischemic heart disease. Am J Physiol Heart Circ Physiol. 2000;278:H741-7.

15. Gilbert NF, Meyer PE, Tauriainen MP, Chao RY, Patel JB, Malloy $\mathrm{CR}$, et al. Effects of hypothermia on myocardial substrate selection. Ann Thorac Surg. 2002;74:1208-12.

16. Reed MK, Barak C, Malloy CR, Maniscalco SP, Jessen ME. Effects of glutamate and aspartate on myocardial substrate oxidation during potassium arrest. J Thorac Cardiovasc Surg. 1996;112:1651-60. 